A $\mathrm{C}$ G $\mathrm{C}$ Rec. Nat. Prod. 13:2 (2019) 136-140

\title{
A New Acylated Benzyl Alcohol Glucoside from
}

\section{Syzygium austroyunnanense}

\author{
Feng Li $\odot$, Shaohua Xu॰, Yan Zhao $\odot$, Mengjia Li $\odot$, Wen Xu॰ \\ Yikao Hu॰, Dingli Zhang $\odot$ and Yong Zhao ${ }^{*}$
}

College of Chemistry and Chemical Engineering, Yunnan Normal University, Kunming 650500, China

(Received April 16, 2018; Revised June 08, 2018; June 21, 2018)

\begin{abstract}
A new acylated benzyl alcohol glucoside, benzyl alcohol 3',6'-di- $O$-galloyl- $\beta$-glucopyranoside (1), together with a known analogue, 6'-O-galloyl- $\beta$-glucopyranoside (2) and a cyanogenic glucoside, 6'- $O$ galloylprunasin (3) were isolated from the leaves of Syzygium austroyunnanense. Their structures were characterized based on the spectroscopic methods and comparison with literature. This is the first phytochemical study on Syzygium austroyunnanense.
\end{abstract}

Keywords: Syzygium austroyunnanense; Myrtaceae; acylated glycoside. (C) 2018 ACG Publications. All rights reserved.

\section{Introduction}

The genus Syzygium (Myrtaceae) comprises about 500 species, mostly grown in the tropic regions of the world [1]. Many of them have been used as edible and medicinal plants in Southeast Asian [2]. Previous research has demonstrated that hydrolysable tannins, flavonoids, chromone derivatives, phenylpropanoids, triterpenes, and phloroglucinols derivatives are the main bioactive constituents in this genus [3-9]. Syzygium austroyunnanense Chang et Miau, called as "Bajiamiao" in the Dai nationality, is a fruit tree which is native to Xishuang Banna Prefecture Yunnan province and Guangxi province in China [10]. To the best of our knowledge, no phytochemical research of this species have been reported so far. In our efforts to search for bioactive constituents, the leaves of $S$. austroyunnanense were investigated and one new acylated glycoside, benzyl 3',6'-di- $O$-galloyl- $\beta$-glucopyranoside (1), together with two known analogues, benzyl 6'-O-galloyl- $\beta$-glucopyranoside (2) and 6'-Ogalloylprunasin (3) were obtained. Here, we present the isolation and characterization of the new compound.

* Corresponding author: E- Mail: zhaooy@126.com

The article was published by ACG Publications

http://www.acgpubs.org/journal/records-of-natural-products (C) March-April 20192018 EISSN:1307-6167 DOI: http://doi.org/10.25135/rnp.85.18.04.277 


\section{Materials and Methods}

UV spectra were acquired in $\mathrm{MeOH}$ with a Shimadzu UV-2401PC UV-vis spectrophotometer. IR spectra were measured on a Bruker Tensor 27 FTIR Spectrometer with KBr disks. NMR spectra were recorded on a Bruker Avance III-600 and a Bruker AM-400 instruments with TMS as internal standard. ESI-MS spectra were recorded on a Waters Xevo TQ-S UPLC Triple Quadrupole Mass Spectrometer. Column chromatography was performed using silica gel (Qingdao Marine Chemical Factory, China, 200-300 mesh), Sephadex LH-20 (Pharmacia Biotech Ltd., Sweden). Thin-layer chromatography (TLC) was performed using precoated silica gel $\mathrm{GF}_{254}$ plates (Qingdao Marine Chemical Factory). Semipreparative HPLC was performed on a Hitachi Chromaster system (Hitachi, Ltd., Japan) equipped with an YMC-Triart $\mathrm{C}_{18}$ column $(250 \mathrm{~mm} \times 10 \mathrm{~mm}$ i.d., $5 \mu \mathrm{m}$, YMC Corporation, Japan), using a flow rate of $3.0 \mathrm{~mL} / \mathrm{min}$ at a column temperature of $25^{\circ} \mathrm{C}$, and detection was performed with a DAD detector.

\subsection{Plant Material}

The leaves of Syzygium austroyunnanense Chang et Miau were collected in October 2014 from Xishuang Banna Tropical Botanical Garden, Yunnan Province, People's Republic of China, and were authenticated by Mr. Yu Chen at the State Key Laboratory of Phytochemistry and Plant Resources in West China, Kunming Institute of Botany, Chinese Academy of Science. Voucher specimens (KIB 20141009) were deposited at Kunming Institute of Botany, Chinese Academy of Science.

\subsection{Extraction and Isolation}

The air-dried and powdered leaves of $S$. austroyunnanense $(2.5 \mathrm{~kg})$ were extracted with $\mathrm{CH}_{3} \mathrm{OH}$ $(6 \mathrm{~L} \times 3)$ at room temperature. The extracts were concentrated by rotary evaporator under reduced pressure to remove organic solvent. The extract $(530 \mathrm{~g})$ was suspended in $\mathrm{H}_{2} \mathrm{O}(0.5 \mathrm{~L})$ and then successively partitioned with petroleum ether $(4 \times 1 \mathrm{~L})$, EtOAc $(4 \times 1 \mathrm{~L})$, and $\mathrm{n}-\mathrm{BuOH}(4 \times 1 \mathrm{~L})$, sequentially. The EtOAc extract ( $42.0 \mathrm{~g}$ ) was subjected to silica gel column chromatography (CC) using a gradient system of petroleum ether (PE)- $\mathrm{Me}_{2} \mathrm{CO}(1: 0-0: 1)$ to afford eight fractions ( $\left.\mathrm{Fr} \mathrm{A}-\mathrm{H}\right)$.

Fraction F (3.5 g) was decolorized on a MCI gel (CHP 20P) CC eluted by $92 \% \mathrm{MeOH}_{-} \mathrm{H}_{2} \mathrm{O}$, and then divided into three subfractions (Fr. F-1-3) by silica gel (200-300 mesh) CC eluting with $\mathrm{CHCl}_{3}-\mathrm{MeOH}$ (8:1). Subfraction Fr. F-1 was further divided into three subfractions (Fr. F-1-1-3) by silica gel CC eluting with $\mathrm{CHCl}_{3}-\mathrm{MeOH}$ (14:1). Fr. F-1-1 was separated by Sephadex LH-20 column

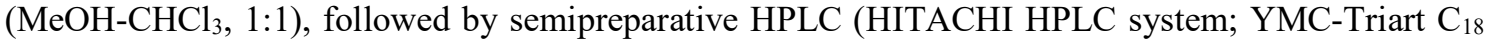
column, $250 \times 10 \mathrm{~mm}$; DAD detector, $\mathrm{MeOH}-\mathrm{H}_{2} \mathrm{O} 35: 65,210 \mathrm{~nm}, 3.0 \mathrm{~mL} / \mathrm{min}$ ) to give compounds 2 $\left(3.5 \mathrm{mg}, \mathrm{t}_{\mathrm{R}}=17.5 \mathrm{~min}\right)$ and $\mathbf{3}\left(21.0 \mathrm{mg}, \mathrm{t}_{\mathrm{R}}=12.5 \mathrm{~min}\right)$. Fr. F-2 was further divided into four subfractions (Fr. F-2-1-4) by silica gel CC eluting with $\mathrm{CHCl}_{3}-\mathrm{MeOH}(10: 1)$. Compound 1 (5.0 mg, $\left.\mathrm{t}_{\mathrm{R}}=21.0 \mathrm{~min}\right)$ was purified from Fr. F-2-4 by Sephadex LH-20 column ( $\left.\mathrm{MeOH}-\mathrm{CHCl}_{3}, 1: 1\right)$, and semipreparative HPLC (HITACHI HPLC system; YMC-Triart $\mathrm{C}_{18}$ column, $250 \times 10 \mathrm{~mm}$; DAD detector, $\mathrm{MeOH}-\mathrm{H}_{2} \mathrm{O}$ $35: 65,210 \mathrm{~nm}, 3.0 \mathrm{~mL} / \mathrm{min})$, sequently.

Benzyl 3',6'-di-O-galloyl- $\beta$-glucopyranoside (1): Amorphous powder; ${ }^{1} \mathrm{H}-\mathrm{NMR}\left(600 \mathrm{MHz}, \mathrm{CD}_{3} \mathrm{OD}\right)$ and ${ }^{13} \mathrm{C}-\mathrm{NMR}\left(150 \mathrm{MHz}, \mathrm{CD}_{3} \mathrm{OD}\right)$ spectral data see Table 1; HR-ESI-MS at $m / z 597.1216[\mathrm{M}+\mathrm{Na}]^{+}$(calcd for $\left.\mathrm{C}_{27} \mathrm{H}_{26} \mathrm{O}_{14}, 597.1215\right)$.

Benzyl 6'-O-galloyl- $\beta$-glucopyranoside (2): Amorphous powder; ${ }^{1} \mathrm{H}-\mathrm{NMR}\left(600 \mathrm{MHz}, \mathrm{CD}_{3} \mathrm{OD}\right)$ and ${ }^{13} \mathrm{C}$ NMR (150 MHz, $\left.\mathrm{CD}_{3} \mathrm{OD}\right)$ spectral data see Table 1.

6'-O-galloylprunasin (3): Amorphous powder; ${ }^{1} \mathrm{H}-\mathrm{NMR}\left(500 \mathrm{MHz}, \mathrm{CD}_{3} \mathrm{OD}\right) \delta \mathrm{H}: 6.70(1 \mathrm{H}, \mathrm{s}, \mathrm{H}-2), 6.87$ $(1 \mathrm{H}, \mathrm{d}, J=8.5 \mathrm{~Hz}, \mathrm{H}-5), 6.71(1 \mathrm{H}, \mathrm{d}, J=8.5 \mathrm{~Hz}, \mathrm{H}-6), 3.35(2 \mathrm{H}, \mathrm{d}, J=8.5 \mathrm{~Hz}, \mathrm{H}-7), 5.97(1 \mathrm{H}, \mathrm{m}, \mathrm{H}-$ 8), $5.10(1 \mathrm{H}, \mathrm{m}, \mathrm{Ha}-9), 5.08(1 \mathrm{H}, \mathrm{m}, \mathrm{Hb}-9), 3.90(3 \mathrm{H}, \mathrm{s}) ;{ }^{13} \mathrm{C}-\mathrm{NMR}(125 \mathrm{MHz}, \mathrm{CD} 3 \mathrm{OD}) \delta \mathrm{C}: 131.9(\mathrm{C}-$ 
1), 111.1 (C-2), 146.4 (C-3), 144.2 (C-4), 114.2 (C-5), 121.2 (C-6), 39.9 (C-7), 137.8 (C-8), 115.5 (C9), $-\mathrm{OCH}_{3}$ (55.9).; ESI-MS at $m / z 421[\mathrm{M}-\mathrm{H}]$.

Table 1. ${ }^{1} \mathrm{H}$ NMR and ${ }^{13} \mathrm{C}$ NMR Data for $\mathbf{1}-\mathbf{2}$ in MeOD at $600 \mathrm{MHz}$ and $150 \mathrm{MHz}$, respectively

\begin{tabular}{|c|c|c|c|c|c|c|c|c|c|c|c|}
\hline \multirow{2}{*}{ NO. } & \multicolumn{2}{|l|}{1} & \multirow{2}{*}{ NO. } & \multicolumn{2}{|c|}{1} & \multirow{2}{*}{ NO. } & \multicolumn{2}{|l|}{2} & \multirow{2}{*}{ NO. } & \multicolumn{2}{|c|}{2} \\
\hline & $\delta_{\mathrm{H}}$ & $\delta_{\mathrm{C}}$ & & $\delta \delta_{\mathrm{H}}$ & $\delta_{C}$ & & $\delta_{\mathrm{H}}$ & $\delta_{\mathrm{C}}$ & & $\delta_{\mathrm{H}}$ & $\delta_{\mathrm{C}}$ \\
\hline 1 & & 140.1 & $1 "$ & & 121.6 & 1 & & 140.1 & $1 "$ & & 121.6 \\
\hline 2 & 7.35 br d (7.3) & 129.5 & $2^{\prime \prime}$ & $7.12 \mathrm{~s}$ & 110.4 & 2 & 7.26 br d (7.2) & 129.5 & $2 "$ & $7.02 \mathrm{~s}$ & 110.3 \\
\hline 3 & $7.29 \mathrm{t}(7.3)$ & 129.5 & $3 "$ & & 146.6 & 3 & $7.18 \mathrm{t}(7.2)$ & 129.6 & $3 "$ & & 146.7 \\
\hline 4 & $7.24 \mathrm{t}(7.3)$ & 129.0 & $4 "$ & & 138.8 & 4 & $7.14 \mathrm{t}(7.2)$ & 128.9 & $4 "$ & & 138.9 \\
\hline 5 & $7.29 \mathrm{t}(7.3)$ & 129.5 & $5^{\prime \prime}$ & & 146.6 & 5 & $7.18 \mathrm{t}(7.2)$ & 129.6 & $5^{\prime \prime}$ & & 146.7 \\
\hline 6 & 7.35 br d (7.3) & 129.5 & 6" & $7.12 \mathrm{~s}$ & 110.4 & 6 & 7.26 br d (7.2) & 129.5 & $6^{\prime \prime}$ & $7.02 \mathrm{~s}$ & 110.3 \\
\hline $7 \mathrm{a}$ & $4.86 \mathrm{~d}(11.8)$ & \multirow{2}{*}{72.1} & $7 "$ & & 168.3 & $7 \mathrm{a}$ & $4.74 \mathrm{~d}(11.8)$ & \multirow{2}{*}{71.8} & $7 "$ & & \multirow[t]{9}{*}{168.5} \\
\hline $7 b$ & $4.66 \mathrm{~d}(11.8)$ & & $1^{\prime \prime \prime}$ & & 121.9 & $7 b$ & $4.53 \mathrm{~d}(11.8)$ & & & & \\
\hline $1^{\prime}$ & $4.50 \mathrm{~d}(7.9)$ & 103.3 & $2^{\prime \prime \prime}$ & $7.13 \mathrm{~s}$ & 110.5 & $1^{\prime}$ & $4.24 \mathrm{~d}(7.7)$ & 103.2 & & & \\
\hline $2^{\prime}$ & $3.53 \mathrm{dd}(9.5,7.9)$ & 73.8 & 3"' & & 146.7 & $2^{\prime}$ & $3.27 \mathrm{t}(9.0)$ & 75.3 & & & \\
\hline $3^{\prime}$ & $5.14 \mathrm{t}(9.2)$ & 79.1 & 4 '" & & 139.9 & $3^{\prime}$ & $3.32 \mathrm{t}(9.2)$ & 78.1 & & & \\
\hline $4^{\prime}$ & $3.68 \mathrm{~m}$ & 70.4 & $5^{\prime \prime \prime}$ & & 146.7 & $4^{\prime}$ & $3.42 \mathrm{~m}$ & 72.0 & & & \\
\hline $5^{\prime}$ & $3.68 \mathrm{~m}$ & 75.7 & 6"' & $7.13 \mathrm{~s}$ & 110.5 & $5^{\prime}$ & $3.54 \mathrm{~m}$ & 75.7 & & & \\
\hline 6'a & $4.58 \mathrm{~d}(11.6)$ & \multirow{2}{*}{64.7} & 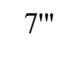 & & 168.4 & 6'a & $4.45 \mathrm{dd}(11.9,1.8)$ & \multirow{2}{*}{64.9} & & & \\
\hline $6 ' b$ & $4.48 \mathrm{~m}$ & & & & & $6 \mathrm{~b}$ & $4.33 \mathrm{dd}(11.9,6.0)$ & & & & \\
\hline
\end{tabular}

\section{Results and Discussion}

Compound 1 was obtained as a lavender amorphous powder. Its molecular formula, $\mathrm{C}_{27} \mathrm{H}_{26} \mathrm{O}_{14}$, was deduced from the HR-ESI-MS $\left(m / z 597.1216[\mathrm{M}+\mathrm{Na}]^{+}\right.$, calcd. 597.1215). Strong UV absorptions at $214 \mathrm{~nm}$ and $276 \mathrm{~nm}$ implied it had large conjugated systems. Its IR spectrum showed absorption bands for conjugated carbonyl groups at $1700 \mathrm{~cm}^{-1}$ and hydroxyl groups at $3397 \mathrm{~cm}^{-1}$. The ${ }^{1} \mathrm{H}$ NMR spectrum

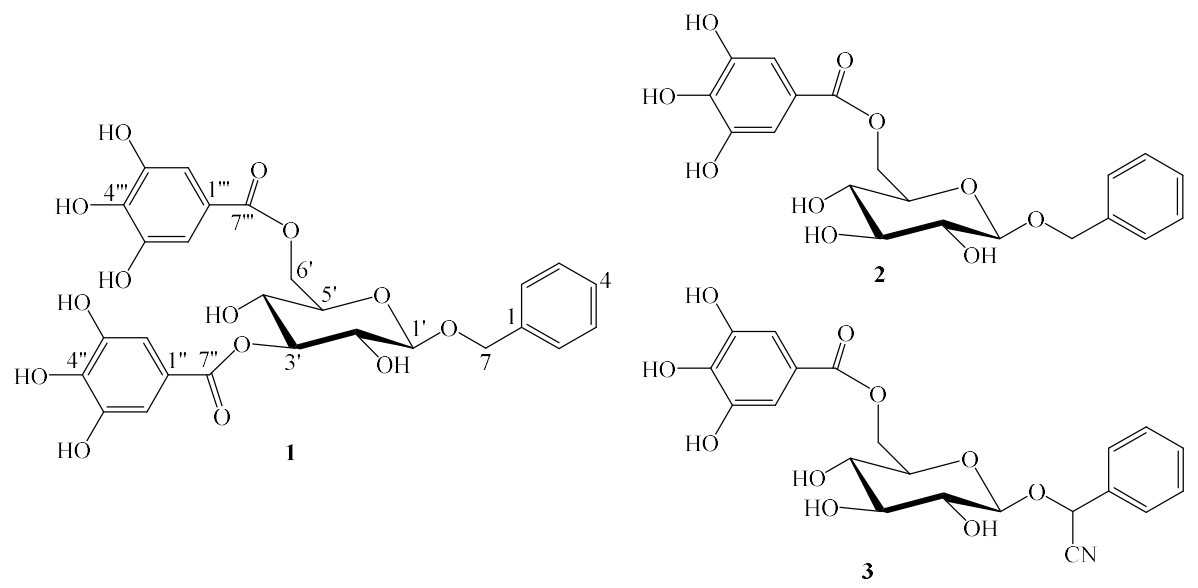

Figure 1. The chemical structures of compounds 1-3 
revealed the presence of two galloyl groups $(\delta 7.12,2 \mathrm{H}, \mathrm{s} ; 7.13,2 \mathrm{H}, \mathrm{s})$, a monosubstituted benzene ring $(\delta 7.35,2 \mathrm{H}, \mathrm{br} \mathrm{d}, J=7.3 \mathrm{~Hz} ; 7.29,2 \mathrm{H}, \mathrm{t}, J=7.3 \mathrm{~Hz} ; 7.24,1 \mathrm{H}, \mathrm{t}, J=7.3 \mathrm{~Hz})$, and signals diagnostic of a ${ }^{4} \mathrm{C}_{1}$-glucopyranosyl residue. An anomeric proton signal of the glucose at $\delta 4.50(\mathrm{~d}, J=7.9 \mathrm{~Hz})$ indicated a $\beta$-configuration for the glucosidic bond. These spectral features along with two carboxyl carbon signals at $\delta 168.3$ and 168.4 in the ${ }^{13} \mathrm{C}$ NMR spectrum suggested that $\mathbf{1}$ is a di-O-galloyl- $\beta$ glucopyranoside. Additionally, a methylene group $\left[\delta_{\mathrm{H}} 4.86,4.66\right.$ (each d, $J=11.8 \mathrm{~Hz}$ ); $\left.\delta_{\mathrm{C}} 72.1\right]$ was evident. The ${ }^{1} \mathrm{H}$ and ${ }^{13} \mathrm{C}$ NMR spectra of $\mathbf{1}$ closely resembled those of a known compound $\mathbf{2}$, benzyl 6'$O$-galloyl- $\beta$-glucopyranoside [11], except that $\mathbf{1}$ had one more galloyl than $\mathbf{2}$. The location of galloyl units at $3^{\prime}-O$ and $6^{\prime}-O$ of the glucopyranose moiety was established by the low-field shifts of the H-3' $\left(\delta_{\mathrm{H}} 5.14\right)$ and $\mathrm{H}-6^{\prime}$ signals $\left(\delta_{\mathrm{H}} 4.58,4.48\right)$, which was proved by the correlations of $\mathrm{H}-3^{\prime} / \mathrm{C}-7^{\prime \prime}$ and $\mathrm{H}-$ $6^{\prime} / \mathrm{C}-7$ "'. The location of benzyl was attached to $\mathrm{C}-1$ ' through $\mathrm{O}$ atom, which was confirmed by the cross peaks from $\mathrm{H}_{2}-7$ to $\mathrm{C}-1$ ' and from $\mathrm{H}_{2}-7$ to $\mathrm{C}-2$ and $\mathrm{C}-6$. Thus, compound 1 was assigned as benzyl 3', 6 '-di- $O$-galloyl- $\beta$-glucopyranoside.

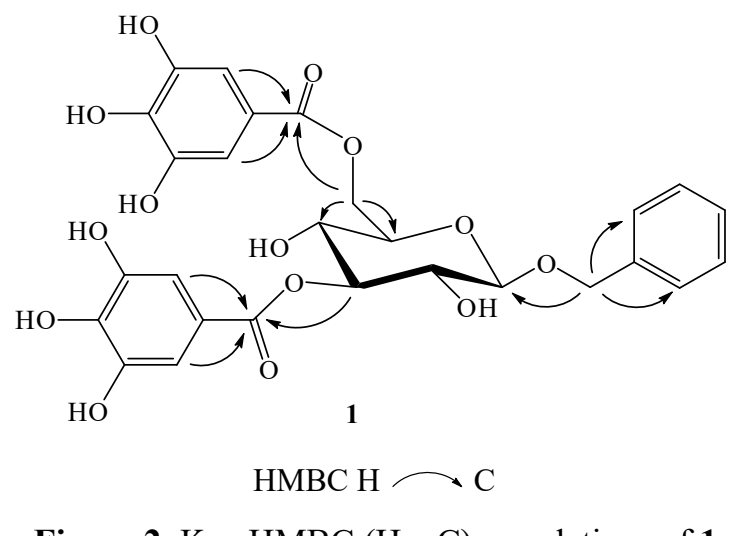

Figure 2. Key $\mathrm{HMBC}(\mathrm{H} \rightarrow \mathrm{C})$ correlations of 1

The known compounds were determined to be benzyl 6'-O-galloyl- $\beta$-glucopyranoside (2) and 6'-O-galloylprunasin (3) [11] by comparing their spectroscopic data with those in the literature.

\section{Acknowledgments}

This work was financially supported by The National Natural Science Foundation of China (No. 31560098) Mid-aged and Young Academic and Technical Leader Raising Foundation of Yunnan Province (No. 2010CI040), China.

\section{Supporting Information}

Supporting information accompanies this paper on http://www.acgpubs.org/journal/recordsof-natural-products

\section{ORCID}

Feng Li: 0000-0002-3943-5058

Shaohua $\mathrm{Xu}$ : 0000-0003-3526-1705

Yan Zhao: 0000-0002-1638-7751

Mengjia Li: $0000-0002-7454-3767$

Wen Xu: $0000-0003-1588-8850$

Yikao Hu: 0000-0003-3913-9408 
Dingli Zhang: 0000-0002-9073-6761

Yong Zhao : $\underline{0000-0002-3996-2480}$

\section{References}

[1] L.W. Tian, M. Xu, D. Wang, H.T. Zhu, C.R. Yang and Y.J. Zhang (2011). Phenolic constituents from the leaves of Syzygium forrestii Merr. and Perry, Biochem. System. Ecol. 39, 156-158.

[2] G.Q. Li, Y.B. Zhang, P. Wu, N.H. Chen, Z.N. Wu, L. Yang, R.X. Qiu, G.C. Wang and Y.L. Li (2015). New phloroglucinol derivatives from the fruit tree Syzygium jambos and their cytotoxic and antioxidant activities, J. Agric. Food Chem. 63, 10257-10262.

[3] T. Manaharan, D. Appleton, H.M. Cheng and U.D. Palanisamy (2012). Flavonoids isolated from Syzygium aqueum leaf extract as potential antihyperglycaemic agents, Food Chem. 132, 1802-1807.

[4] M.N. Samy, S. Sugimoto, K. Matsunami, H. Otsuka and M.S. Kamel (2014). One new flavonoid xyloside and one new natural triterpene rhamnoside from the leaves of Syzygium grande, Phytochem. Lett. 10, 8690.

[5] M. Miyazawa and M. Hisama (2003). Antimutagenic activity of phenylpropanoids from clove (Syzygium aromaticum), J. Agric. Food Chem. 51, 6413-6422.

[6] F.F. Liu, T. Yuan, W. Liu, H. Ma, N.P. Seeram, Y.Y. Li, L. Xu, Y. Mu, X.S. Huang and Y.L. Li (2017). Phloroglucinol derivatives with protein tyrosine phosphatase 1B inhibitory activities from Eugenia jambolana seeds, J. Nat. Prod. 80, 544-550.

[7] T. Manaharan, H.M. Cheng and U.D. Palanisamy (2013). Syzygium aqueum leaf extract and its bioactive compounds enhances pre-adipocyte differentiation and 2-NBDG uptake in 3T3-L1cells, Food Chem. 136, 354-363.

[8] R. Omar, L.Y. Li, T. Yuan and N.P. Seeram (2012). $\alpha$-Glucosidase inhibitory hydrolysable tannins from Eugenia jambolana seeds, J. Nat. Prod. 75, 1505-1509.

[9] N. Yao, W. Yao, J.X. Lei and K.W. Wang (2013). Lignans from Syzygium grijsii and their chemotaxonomic significance, Biochem. System. Ecol. 46, 79-82.

[10] J. Chen (1984). Flora of China. Vol 53 (1). Beijing: Science Press, pp. 72.10

[11] J.H. Isaza, H. Ito and T. Yoshida (2001). A flavonol glycoside-lignan ester and accompanying acylated glucosides from Monochaetum multiflorum, Phytochemistry 58, 321-327.

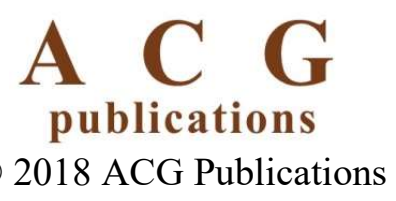

